\title{
Free and Forced Vibration Analyses of Hyperbolic Cooling Tower Shell Using Harmonic Solid Ring Finite Elements
}

\author{
Ali I. KARAKAS \\ Karadeniz Technical University, Department of Civil Engineering, Trabzon, Turkey \\ Ayse T. DALOGLU* \\ Karadeniz Technical University, Department of Civil Engineering, Trabzon, Turkey
}

\begin{tabular}{ll}
\hline \hline Article history & This paper discusses free and forced vibration responses of \\
Received: & hyperbolic cooling tower shell which is one of the complex real life \\
& applications of axisymmetric structures. 9-noded harmonic solid \\
Received in revised form: & ring finite element is used in the numerical model of the cooling \\
01.06 .2015 & tower. Physically a three-dimensional cooling tower problem is \\
Accepted: & reduced to a two dimensional one by expressing earthquake loading \\
01.06 .2015 & in the form of Fourier series for a single harmonic with the help of \\
Key words: & harmonic elements. Therefore, the complete solution for the \\
\hline Vibration analysis, cooling & problem is obtained simply for a single load component that makes \\
tower shell, harmonic solid ring & the model computationally much more efficient. A computer \\
finite element & program is coded in Matlab for the purpose and the results obtained \\
& in the study are verified with the results available in the literature. \\
& A parametric study is also performed for the variations in shell \\
& curvature of the tower. The time history analysis method is used for \\
& the dynamic response of the cooling tower shell. Acceleration \\
& records of Düzce earthquake is used in the study and the results are \\
& presented in tabular and graphical formats comparatively for \\
& varying shell curvature. It can be concluded that circumferential \\
& mode number and shell curvature have significant effects on the \\
& dynamic responses of cooling towers. \\
\hline \hline
\end{tabular}

\footnotetext{
* Correspondence: Prof. Dr. Ayşe T. Daloglu, Karadeniz Technical University, Faculty of Engineering, Civil Engineering Dept., 61080 Trabzon, Turkey. e-mail :aysed@ktu.edu.tr Phone : (+90)-462-377-2662, fax: (+90)-462-377-2606
} 


\section{Introduction}

One of the thin-walled slender structures in civil engineering is reinforced concrete cooling tower which is the essential component of thermal and nuclear power stations to reduce the temperature of the cooling water-circuit. Such shell structures are exposed to various loadings such as selfweight, wind and earthquake loads. In the design process of such structures free vibration characteristics and seismic responses play an important role especially when they are constructed around the region prone to severe earthquakes. Therefore, it is especially necessary and urgent to know the seismic behavior of the huge towers under severe earthquake attacks. The loads applied to the shell wall of a cooling tower due to design earthquake can be determined by the response spectrum or time history analysis. The former and the later method require a free vibration analysis and ground motion records, respectively.

The dynamic analysis of cooling tower structures is studied by researchers who are aware of the importance of earthquake loading on such important structures in high seismic zones. For example, Hashish and Abu-Sitta [1], Prasahanth and Sayeed [2] and Nasir et al. [3] , Sam-Young and Sang-Yun [4,5] and Calladine [6] presented the effects of some tower geometrical parameters on the frequency response of cooling towers fixed or simply supported at the shell base. Moreover, Tande and Chougule [7] and

$$
\{u(r, \theta, z)\}=\left\{\begin{array}{l}
u_{r} \\
u_{\theta} \\
u_{z}
\end{array}\right\}=\left\{\begin{array}{l}
\bar{u}_{r}+\overline{\bar{u}}_{r} \\
\bar{u}_{\theta}+\overline{\bar{u}}_{\theta} \\
\bar{u}_{z}+\overline{\bar{u}}_{z}
\end{array}\right\}=\left\{\begin{array}{l}
\sum_{m=0}^{\infty} \bar{u}_{r m} \cos m \theta+\sum_{m=0}^{\infty} \bar{u}_{r m} \sin m \theta \\
\sum_{m=0}^{\infty} \bar{u}_{\theta m} \sin m \theta-\sum_{m=0}^{\infty} \bar{u}_{\theta m} \cos m \theta \\
\sum_{m=0}^{\infty} \bar{u}_{z m} \cos m \theta+\sum_{m=0}^{\infty} \bar{u}_{z m} \sin m \theta
\end{array}\right\}
$$

where $m$ is the circumferential mode (harmonic) number and symbols $u_{r}, u_{\theta}$ and $u_{z}$ indicate the radial, circumferential and
Kulkarni and Kulkarni [8] carried out static and dynamic analyses of hyperbolic cooling towers fixed at shell base. In all of these studies three-dimensional isotropic shell elements were used to model the shell wall since they consider the problem as three dimensional in analysis. This approach is also a time consuming process since a complete dynamic analysis of such structures requires very large number of analysis.

However, in this study with the help of harmonic analysis physically three dimensional problem is reduced to a two dimensional problem. The solution for the problem is obtained using 9-noded (Ring9) solid quadrilateral ring harmonic elements. So the computational effort significantly decreases for the structural analysis of cooling tower shells. A computer program is coded in Matlab for this purpose and results obtained are verified with the ones available in literature. Later the dynamic analysis of hyperbolic cooling tower shells is performed under earthquake loading. Free vibration analyses are also studied for different hyperbolic curvatures. The results are presented in graphical and tabular formats.

\section{Harmonic Finite Element Model}

For harmonic model in linear elastic analysis, it is possible to demonstrate both the loads and the displacement as Fourier series expansions in terms of circumferential coordinate $\theta[9]$ :

axial displacement components, respectively, see Figure 1. All barred quantities in Eq.(1) are amplitudes approximated using the finite element method, which are functions of $r, z$ 
but not of $\theta$. This produces a harmonic finite element in the (r, z) plane. Single and double barred amplitudes represent symmetric $(f(\theta)=f(-\theta)) \quad$ and anti-symmetric $(f(\theta)=-f(-\theta)) \quad$ displacement terms, respectively. The amplitudes of the displacement components in Eq. (1) can be interpolated from nodal amplitudes using the shape functions. Figure 1 shows the shape and node numbering of the 9-noded ring element as well as coordinate system.

In this study the formulation of symmetric part of the harmonic ring finite element is presented for the simplicity and a detailed explanation and anti-symmetric formulation can be found in [10]. The vector of displacement field within the element can be described in the following form that subscript im specifies that amplitude refers to node $i$ and harmonic $m$ [11]

$$
\{\bar{u}\}=\left\{\begin{array}{l}
\bar{u}_{r}(r, \theta, z) \\
\bar{u}_{\theta}(r, \theta, z) \\
\bar{u}_{z}(r, \theta, z)
\end{array}\right\}=\sum_{m=0}^{\infty}\left(\left[\overline{g_{\theta m}}\right]_{u}[N]\left\{\left\{\bar{d}_{m}\right\}\right)\right.
$$

where $\{\bar{d}\}_{m}$ is the nodal displacement vector for the Fourier term (mode) $m$ and $[N]$ is the shape functions matrix, which are defined for a biquadratic ring element (Ring9) used in the present study as written by

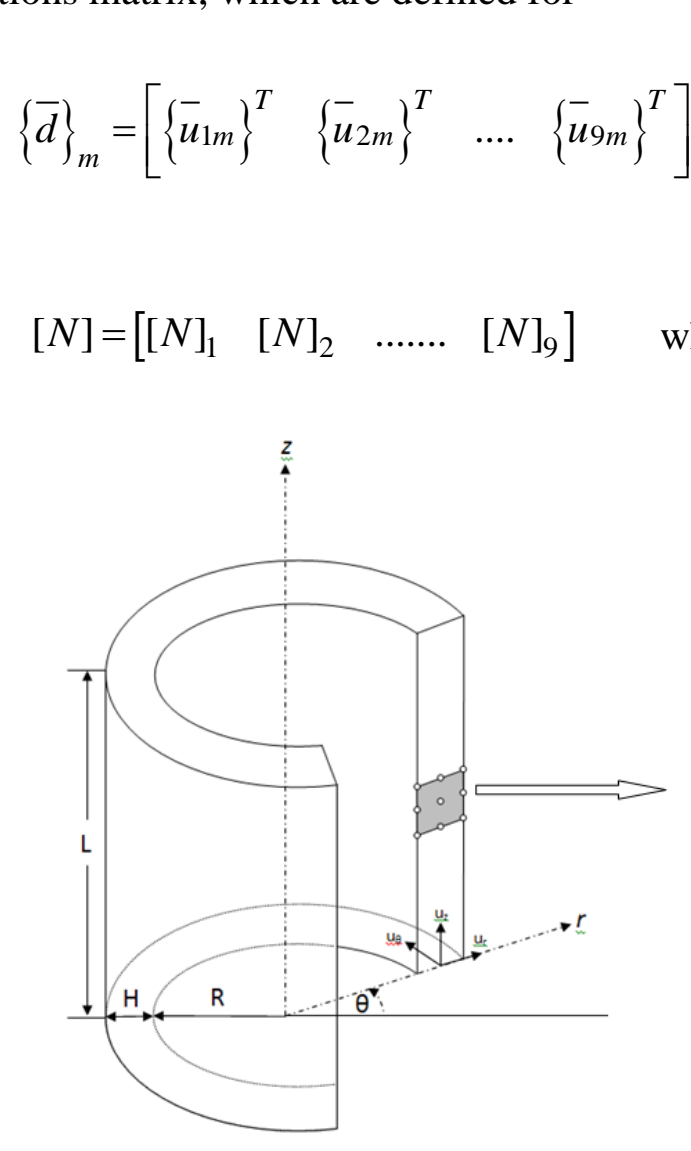

(a)

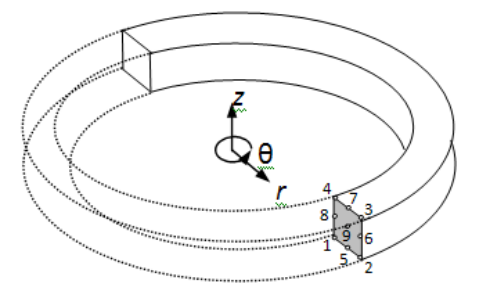

(b)

Figure 1.(a) Displacement components in cylindrical system (b) a solid ring finite element.

The shape function components at each node natural coordinate system are expressed as $i$ for the 9-noded ring element in terms of [12]: 


$$
\begin{array}{rlrl}
N_{i} & =\frac{1}{4} \xi \eta\left[\left(\xi+\xi_{i}\right)\left(\eta+\eta_{i}\right)\right. & & \text { for } \mathrm{i}=1,2,3,4 \\
N_{i} & =\frac{1}{2} \eta\left(1-\xi_{i} \xi^{2}\right)\left(\eta+\eta_{i}\right) & & \text { for } \mathrm{i}=5,7 \\
N_{i} & =\frac{1}{2} \xi\left(\xi+\xi_{i}\right)\left(1-\eta_{i} \eta^{2}\right) & \text { for } \mathrm{i}=6,8 \\
N_{9} & =\left(1-\xi^{2}\right)\left(1-\eta^{2}\right) &
\end{array}
$$

where $\xi_{i}=(-1,1,1,-1,1,1,1,-1)$ and $\eta_{i}=(-1,-1,1,1,-1,1,1,1)$.
Also, the matrices of harmonic functions for harmonic $m$ are:

$$
\left[\overline{g_{\theta m}}\right]_{u}=\left[\begin{array}{ccc}
\cos m \theta & 0 & 0 \\
0 & \sin m \theta & 0 \\
0 & 0 & \cos m \theta
\end{array}\right]
$$

Strains and stresses in an element can also be Fourier term $m$, the strain vector in stated in terms of the Fourier series. For a cylindrical coordinates can be written as:

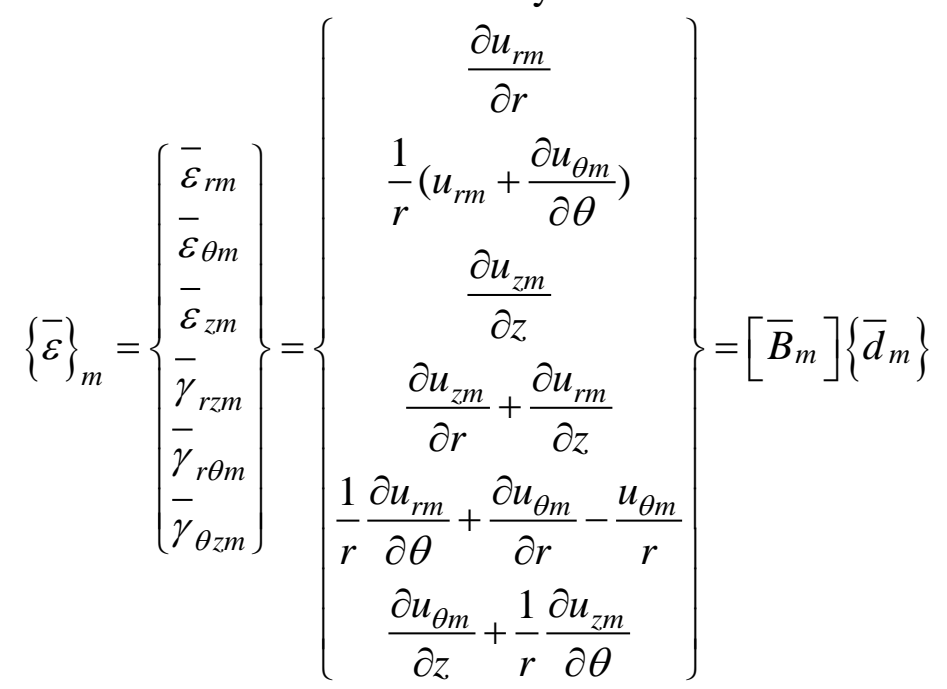

The strain displacement matrices can be stated as follows for harmonic $m$ :

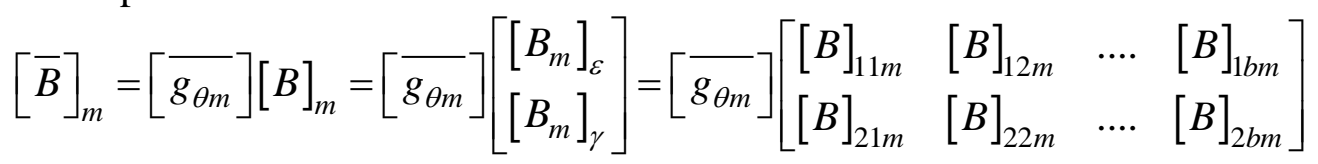

where $\left[\bar{B}_{m}\right]$ is the matrix which relates the $\left[\overline{g_{\theta m}}\right]$ of the harmonic functions for the symmetric nodal displacement amplitudes harmonic $m$ is:

with corresponding strains and the matrix

$$
\left[\overline{g_{\theta m}}\right]=\left[\begin{array}{cc}
{\left[\overline{g_{\theta m}}\right]_{\varepsilon}} & 0 \\
0 & {\left[\overline{g_{\theta m}}\right]_{\gamma}}
\end{array}\right]
$$

where

$$
\left[\overline{g_{\theta m}}\right]_{\varepsilon}=\cos m \theta\left[\begin{array}{lll}
1 & 0 & 0 \\
0 & 1 & 0 \\
0 & 0 & 1
\end{array}\right] \text { and }\left[\overline{g_{\theta m}}\right]_{\gamma}=\left[\begin{array}{ccc}
\cos m \theta & 0 & 0 \\
0 & \sin m \theta & 0 \\
0 & 0 & \sin m \theta
\end{array}\right]
$$


and for the $i^{\text {th }}$ node, the submatrices are given as:

$$
\begin{gathered}
{[B]_{1 i m}=\left[\begin{array}{ccc}
N_{i, r} & 0 & 0 \\
\frac{N_{i}}{r} & \frac{m N_{i}}{r} & 0 \\
0 & 0 & N_{i, z}
\end{array}\right]} \\
{[B]_{2 i m}=\left[\begin{array}{ccc}
N_{i, z} & 0 & N_{i, r} \\
-\frac{m N_{i}}{r} & \left(N_{i, r}-\frac{N_{i}}{r}\right) & 0 \\
0 & N_{j, z} & -\frac{m N_{i}}{r}
\end{array}\right]}
\end{gathered}
$$

The stress vector for the $m^{\text {th }}$ harmonic in the equations is given for an isotropic material as cylindrical coordinate system related to the follows [13]:

strain vector through the constitutive

$$
\{\bar{\sigma}\}_{m}=[D]\{\bar{\varepsilon}\}_{m}
$$

in which $[D]$ is the material property matrix equation where $E$ modulus of elasticity and $v$ for isotropic materials given by the following is Poisson's ratio.

$[D]=\left[\begin{array}{cc}{\left[E_{\varepsilon}\right]} & 0 \\ 0 & {\left[E_{\gamma}\right]}\end{array}\right] \quad, \quad\left[E_{\varepsilon}\right]=\frac{E}{(1+v)(1-2 v)}\left[\begin{array}{ccc}1-v & v & v \\ v & 1-v & v \\ v & v & 1-v\end{array}\right] \quad, \quad\left[E_{\gamma}\right]=\frac{E}{2(1+v)}$

\section{Finite Element Matrices}

\subsection{The element stiffness matrix}

The stiffness matrix of a linear system is calculated from the derivation of

$$
U_{e}=\frac{1}{2} \int\{\varepsilon\}^{T}\{\sigma\} d V=\frac{1}{2}\{d\}^{T}[k]\{d\}
$$

Substituting Eqs. (7) and (18) into Eq. (21) for single barred terms the stiffness matrix

$$
\left[\bar{k}_{m}\right]=\iint\left[B_{m}\right]^{T}\left(\int _ { 0 } ^ { 2 \pi } \left[\begin{array}{c}
{\left[\overline{g_{\theta m}}\right]_{\varepsilon}^{T}\left[E_{\varepsilon}\right]\left[\overline{g_{\theta m}}\right]_{\varepsilon}} \\
0
\end{array}\right.\right.
$$

It can be observed that each term in the products of $\left[\overline{g_{\theta m}}\right]_{\varepsilon}^{T}\left[E_{\varepsilon}\right]\left[\overline{g_{\theta m}}\right]_{\varepsilon}$,

$\left[\overline{g_{\theta m}}\right]_{\gamma}^{T}\left[E_{\gamma}\right]\left[\overline{g_{\theta m}}\right]_{\gamma}$ will be a function of $(E, v)$ multiplied by either $\cos ^{2} m \theta$ or $\sin ^{2} m \theta$. Thus, integration over the circumferential direction $\theta$ can be carried out the strain energy of an axisymmetric solid ring element [14].The element strain energy is given as: for symmetric terms in Fourier series expansion is obtained as:

$\left.\left[\overline{g_{\theta m}}\right]_{\gamma}^{T}\left[E_{\gamma}\right]\left[\overline{g_{\theta m}}\right]_{\gamma}\right] d \theta\left[B_{m}\right] r d r d z$

explicitly. The stiffness matrix of a quadrilateral ring element can be numerically integrated by Gauss quadrature rule which is suitable for the finite element applications.

By taking explicit integrations and using numerical integration the stiffness matrices for symmetric terms are calculated from the following expressions which are used this study: 


$$
\begin{array}{r}
\text { for } m=0\left[\bar{k}_{0}\right]=2 \pi\left(\begin{array}{l}
\sum_{k=1}^{p} \sum_{l=1}^{p} w_{k} w_{l}\left[B_{0}\right]_{\varepsilon}^{T}\left(\xi_{k}, \eta_{l}\right)\left[\bar{E}_{\varepsilon o}\right]\left[B_{0}\right]_{\varepsilon}\left(\xi_{k}, \eta_{l}\right) r\left(\xi_{k}, \eta_{l}\right) J\left(\xi_{k}, \eta_{l}\right) \\
+\sum_{k=1}^{p} \sum_{l=1}^{p} w_{k} w_{l}\left[B_{0}\right]_{\gamma}^{T}\left(\xi_{k}, \eta_{l}\right)\left[\bar{E}_{\gamma 0}\right]\left[B_{0}\right]_{\gamma}\left(\xi_{k}, \eta_{l}\right) r\left(\xi_{k}, \eta_{l}\right) J\left(\xi_{k}, \eta_{l}\right)
\end{array}\right) \\
\text { for } m>0\left[\bar{k}_{m}\right]=\pi\left(\begin{array}{l}
\sum_{k=1}^{p} \sum_{l=1}^{p} w_{k} w_{l}\left[B_{m}\right]_{\varepsilon}^{T}\left(\xi_{k}, \eta_{l}\right)\left[E_{\varepsilon}\right]\left[B_{m}\right]_{\varepsilon}\left(\xi_{k}, \eta_{l}\right) r\left(\xi_{k}, \eta_{l}\right) J\left(\xi_{k}, \eta_{l}\right) \\
+\sum_{k=1}^{p} \sum_{l=1}^{p} w_{k} w_{l}\left[B_{m}\right]_{\gamma}^{T}\left(\xi_{k}, \eta_{l}\right)\left[E_{\gamma}\right]\left[B_{m}\right]_{\gamma}\left(\xi_{k}, \eta_{l}\right) r\left(\xi_{k}, \eta_{l}\right) J\left(\xi_{k}, \eta_{l}\right)
\end{array}\right)
\end{array}
$$

where $\xi_{k}$ and $\eta_{l}$ are the Gauss points abscissae whereas $w_{k}$ and $w_{l}$ are the corresponding integration weights, with indices $k$ and $l$ running from 1 to number of Gauss points used. Also $\left[B_{m}\right]_{\varepsilon}\left(\xi_{k}, \eta_{l}\right)$ and $\left[B_{m}\right]_{\gamma}\left(\xi_{k}, \eta_{l}\right)$ mean that these matrices are evaluated at Gauss points; likewise for $r\left(\xi_{k}, \eta_{l}\right)$, the radius of the Gauss point and $J\left(\xi_{k}, \eta_{l}\right)$, the Jacobian determinant that maps the area element in global coordinates $(r, z)$ to area in the natural coordinates $(\xi, \eta)$. The Gauss points numbers used in this study is $p=3$ for full integration.

\subsection{The element mass matrix}

The consistent mass matrix of a harmonic element is calculated from the derivation of the kinetic energy as explained in [14]. The element mass matrices are obtained for the single barred symmetric terms as the followings:

$$
\begin{aligned}
\text { for } m=0 \quad\left[\bar{m}_{0}\right]= & 2 \pi \sum_{k=1}^{p} \sum_{l=1}^{p} w_{k} w_{l} \rho N^{T}\left(\xi_{k}, \eta_{l}\right)\left[\begin{array}{lll}
1 & 0 & 0 \\
0 & 0 & 0 \\
0 & 0 & 1
\end{array}\right] N\left(\xi_{k}, \eta_{l}\right) r\left(\xi_{k}, \eta_{l}\right) J\left(\xi_{k}, \eta_{l}\right) \\
\text { for } m>0 & {\left[\bar{m}_{m}\right]=\pi \sum_{k=1}^{p} \sum_{l=1}^{p} w_{k} w_{l} \rho N^{T}\left(\xi_{k}, \eta_{l}\right) N\left(\xi_{k}, \eta_{l}\right) r\left(\xi_{k}, \eta_{l}\right) J\left(\xi_{k}, \eta_{l}\right) }
\end{aligned}
$$

where $\rho$ is the density of the material.

\section{Equation of motions}

In the present study free and seismically forced vibration behaviors of cooling towers are under consideration.
Therefore, the equation of motion to be solved for free and undamped vibration is:

$$
[\bar{M}]\{\overline{\ddot{u}}\}+[\bar{K}]\{\bar{u}\}=0
$$

where $\{\bar{u}\}$ and $\{\overline{\ddot{u}}\}$ are the global displacement and acceleration vectors and $[\bar{M}]$ and $[\bar{K}]$ are the system mass and stiffness matrices for symmetric terms, respectively. By assuming all points in the system undergo simple harmonic motion with the corresponding natural frequency of $\omega$ when vibrating a nontrivial solution is obtained as [15]: 


$$
\operatorname{det}\left([\bar{K}]-\omega^{2}[\bar{M}]\right)=0
$$

The solution of Eq. (22) is obtained using QR The time dependent undamped equation of inverse iteration method as explained in [12] motion to be solved for seismic inertia forces which produces square of the natural angular is expressed as [16]:

frequencies of the system.

$$
[\bar{M}]\{\overline{\ddot{u}}(t)\}+[\bar{K}]\{\bar{u}(t)\}=-[\bar{M}]\left\{\ddot{u}_{g}(t)\right\}
$$

where $\left\{\ddot{u}_{g}(t)\right\}$ is the ground acceleration vector at time $t$. Newmark direct time integration method is used for the solution of this equation.

\section{Numerical Examples}

Free vibration and seismic responses of a hyperbolic cooling tower are investigated. Additionally, a parametric study is conducted on the cooling tower to investigate the effect of meridional curvature on the free vibration and seismic responses of the tower. For the finite element discretization of the tower 9-noded harmonic solid ring elements are used.

\subsection{Geometry and Material Properties of the Cooling Tower}

The equation of hyperbolic curve of the cooling tower is obtained by Eq. (24) since the hyperbolic curve has double curvature meeting at the throat the characteristic dimension is evaluated for the upper and lower portions of the curve as $b=90.07 \mathrm{~m}$ and $b=68.68 \mathrm{~m}$, by substituting the base $\left(\mathrm{d}_{\mathrm{U}}, \mathrm{Z}_{\mathrm{U}}\right)$ and the top $\left(\mathrm{d}_{\mathrm{H}}, \mathrm{Z}_{\mathrm{H}}\right)$ coordinates in Eq. (25) and (26), respectively [17]. $Z$ coordinate is measured from the throat level. All dimensions in the $R-Z$ plane are specified on the middle surface of the shell wall.

$$
4 R^{2} / d_{T}^{2}-Z^{2} / b^{2}=1
$$

where $b$ is calculated as for upper curve

$$
b=d_{T} Z_{H} / \sqrt{\left(d_{H}^{2}-d_{T}^{2}\right)}
$$

and for lower curve.

$$
b=d_{T} Z_{U} / \sqrt{\left(d_{U}^{2}-d_{T}^{2}\right)}
$$

A constant shell-wall thickness of $240 \mathrm{~mm}$, model. The geometry of the hyperbolic and reinforced concrete with Poisson's ratio cooling tower are depicted in Figure 2 and of 0.2 and elastic modulus of $39 \mathrm{GPa}$ are the geometrical values are presented in Table considered for the finite element numerical 1.

Table 1. Geometric details of hyperbolic cooling tower

\begin{tabular}{lcc}
\hline Description & Symbol & Value $(\mathrm{m})$ \\
\hline Height above throat level & $Z_{H}$ & 25.90 \\
Height below throat level & $Z_{U}$ & 95.60 \\
Top diameter & $d_{H}$ & 58.04 \\
Throat diameter & $d_{T}$ & 55.78 \\
Shell base diameter & $d_{U}$ & 90.60 \\
Wall thickness & $t$ & 0.24 \\
\hline
\end{tabular}




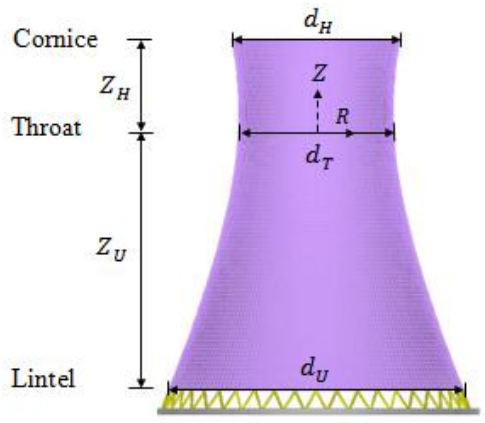

Figure 2. Geometry of the hyperbolic cooling tower

The parameter $\mathrm{K}$ is the indicator of the indicates a more marked curvature of the deviation of the profile from the cylinder meridian. case $(\mathrm{K}=1) . a$ is the throat radius. A larger $\mathrm{K}$

$$
\mathrm{K}=\left(1+\frac{a^{2}}{b^{2}}\right)
$$

\subsection{Earthquake Loading}

Possible loading conditions are dead load, wind load, earthquake load, temperature variations, construction loads, and settlement for a hyperbolic cooling tower [17]. In the scope of this study only earthquake loads are considered. Ground motions generate the earthquake loading on hyperbolic cooling towers. The circumferential responses become axisymmetric $(m=0)$ for uniform vertical ground motion and anti-symmetric $(m=1)$ for uniform horizontal motion. The magnitude of earthquake forces is a function of the mass of the shell and the ground acceleration. A time history finite element analysis is conducted



(a) for time history acceleration records using Newmark direct integration methods. Seismic responses of the hyperbolic cooling tower are investigated for acceleration-time records of Düzce earthquake as plotted in Figure 3(a). The Düzce earthquake occurred on $12^{\text {th }}$ November, 1999 in Turkey, and had the peak accelerations between 5.-10. seconds of the record as seen from Figure 3 (a). The maximum acceleration is $3.73 \mathrm{~m} / \mathrm{s}^{2}$ in the East-West direction. The time step of the record is $0.005 \mathrm{~s}$. Since the dynamic analysis is time consuming for small time steps the duration between 5-10 seconds of the complete record is considered shown in Figure 3(b).



Figure 3. Acceleration-time history record of the Düzce earthquake a) complete b) considered 
duration

\subsection{Free Vibration Analysis of the Cooling Tower}

First of all, the finite element model is verified with the first five circumferential mode and the first lateral mode periods of the cooling tower obtained by Nasir et al. [3].The periods are given in Table 2. The small relative errors indicate that the model produces acceptable results.

Table 2. Finite element model verification; comparison of present results with those from previously established solutions

\begin{tabular}{lcccccc}
\hline \multirow{2}{*}{ Method of solution } & \multicolumn{7}{c}{ Periods of vibration (s) } \\
\cline { 2 - 7 } & 1 & 2 & 3 & 4 & 5 & 6 (lateral) \\
\hline Nasir et al. & 0.723 & 0.666 & 0.662 & 0.593 & 0.549 & 0.294 \\
Present study & 0.729 & 0.671 & 0.669 & 0.597 & 0.553 & 0.294 \\
Relative error $(\%)$ & 0.82 & 0.75 & 1.00 & 0.67 & 0.72 & 0 \\
\hline
\end{tabular}

After verification the natural frequencies and associated mode shapes of the tower are presented conducting a free vibration analysis and a parametric study is conducted to investigate the influence of shape parameter, $\mathrm{K}$ or meridional curvature. A convergence study is carried out to determine the required number of elements to provide an acceptable level of accuracy in the modal analysis. From convergence study 40 Ring9 elements seemed to be good enough in axial direction and one in radial direction for the modal analysis of the cooling tower. Natural frequencies for circumferential modes of $\mathrm{m}=0,1,2, \ldots, 10$ and meridional or longitudinal modes of $n=1,2, \ldots, 5$ for a fixed shell base boundary condition are presented in Table 3 .

Table 3. Natural frequencies of the cooling tower (n:meridional mode, $m$ :circumferential mode)

\begin{tabular}{cccccc}
\hline & \multicolumn{5}{c}{ Natural frequency $(\mathrm{Hz})$} \\
\cline { 2 - 6 }$m$ & \multicolumn{5}{c}{$\mathrm{n}$} \\
\cline { 2 - 6 } & 1 & 2 & 3 & 4 & 5 \\
\hline 0 & 6,67 & 8,59 & 14,29 & 14,99 & 15,74 \\
1 & 3,40 & 7,98 & 12,79 & 14,13 & 14,91 \\
2 & 1,80 & 3,89 & 7,73 & 10,95 & 12,93 \\
3 & 1,48 & 2,03 & 4,64 & 7,47 & 9,97 \\
4 & 1,37 & 1,67 & 2,93 & 5,11 & 7,40 \\
5 & 1,49 & 1,93 & 2,30 & 3,66 & 5,56 \\
6 & 1,85 & 2,13 & 2,67 & 2,97 & 4,41 \\
7 & 2,16 & 2,70 & 2,97 & 3,30 & 3,90 \\
8 & 2,49 & 3,18 & 3,63 & 3,97 & 4,08 \\
9 & 2,87 & 3,64 & 4,39 & 4,55 & 4,96 \\
10 & 3,30 & 4,14 & 4,95 & 5,55 & 5,65 \\
\hline
\end{tabular}

which the first mode is the circumferential

The first five significant circumferential modes $(m)$ are in the order of $4(n=1), 3,5$, $4(\mathrm{n}=2)$, and 2 as given in Table 3. Therefore, it can be concluded that the behavior of the cooling tower is not a beam-like structure in mode of $m=1$ leading to the moving of the cross section about an axis. Also, contraction, expansion, and torsional mode shape frequencies for $m=0$ are the largest 
frequencies as given in Table 3. The curves on Figure 4 for the first 10 harmonics reflect an interesting behavior such that frequencies decrease with increasing circumferential mode number, $m$ until a minimum value is reached hereafter they increase.

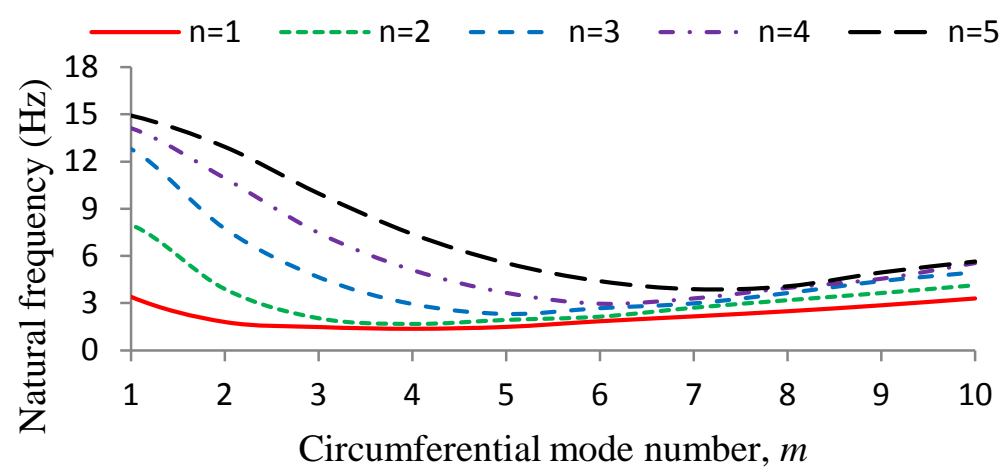

Figure 4. Natural frequencies with respect to circumferential mode number

Representative circumferential mode shapes are shown in Figure 5. The $m=0$ mode represents uniform expansion or contraction of the circumference as shown in Figure 5(a), while $m=1$ corresponds to a beam-like bending with net translation. The higher modes $(m>1) \quad$ generates fluctuating

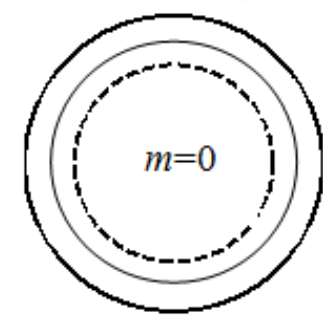

(a)

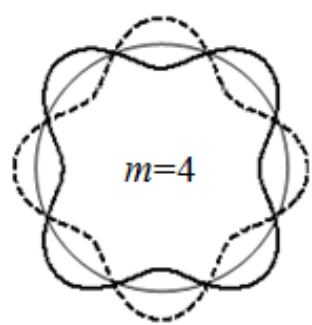

(e)



(b)

displacements around the cross section as shown in Figure 5(c, d, e, f, g, h). These modes appear mostly in thin shell structures. Therefore, for the earthquake analysis only the first mode $(m=1)$ is considered for the uniform horizontal ground motion.

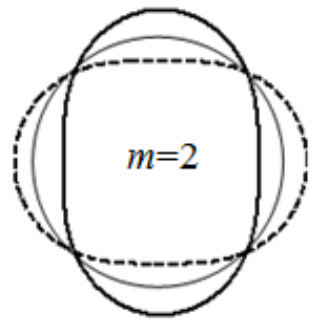

(c)

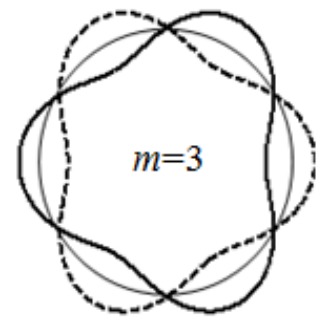

(d)



(f)

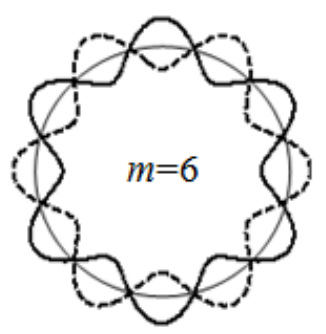

(g)



(h)

Figure 5. Circumferential mode shapes for a) $m=0$ b) $m=1$ c) $m=2$ d) $m=3$ e) $m=4$ f) $m=5$

g) $m=6$ h) $m=7$

Figure 6 depicts the first three meridional or longitudinal mode shapes along the $z$ axis $(\mathrm{n}=1,2,3) \quad$ corresponding to eight circumferential modes $(m=1,2,3,4,5,6,7,8)$. Also, normalized radial amplitudes of vibration are shown in Figure 6. It can be easily seen that the meridional mode shapes $(n=1,2,3)$ for circumferential mode shape $(m=1)$ are similar to the first three mode shapes of a cantilever beam. 


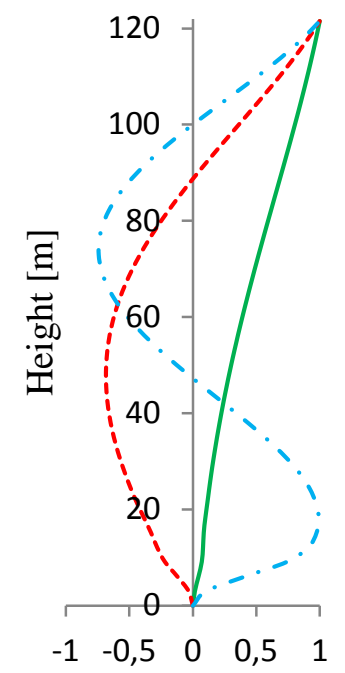

(a)

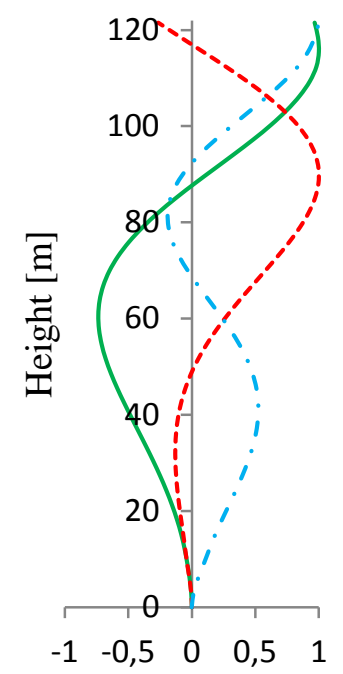

(e)

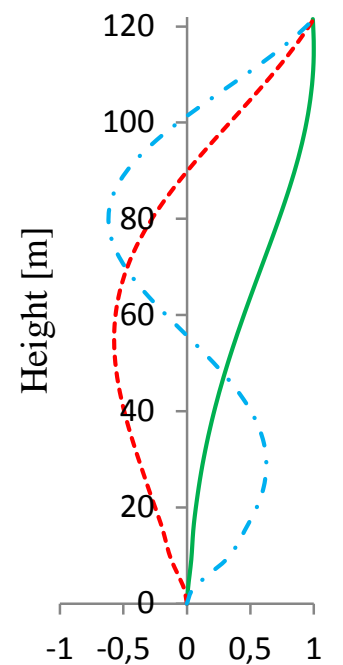

(b)

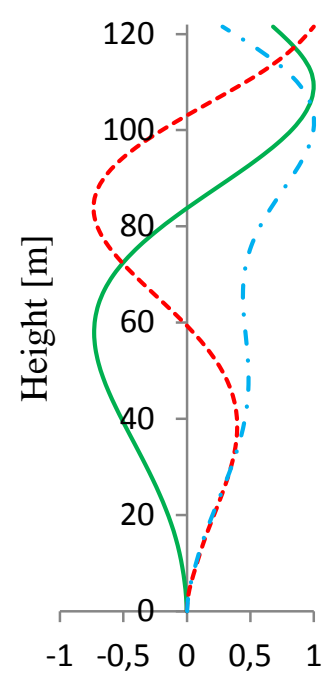

(f)

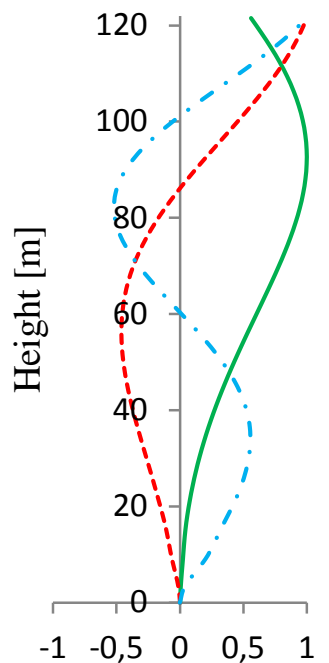

(c)

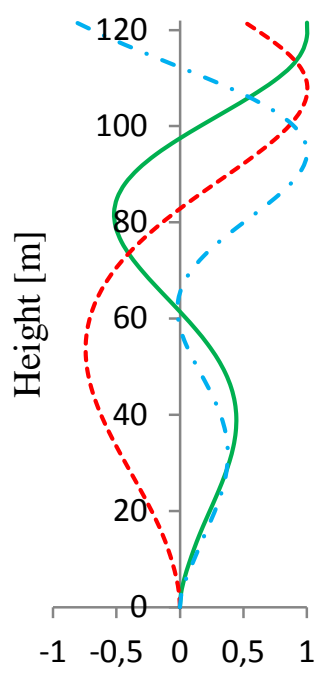

(g)

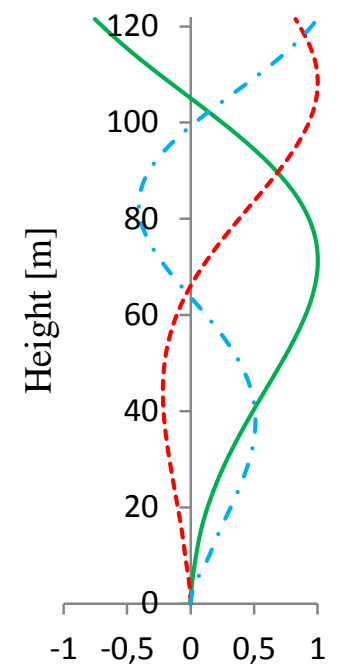

(d)



(h)

Normalized radial amplitudes of vibration

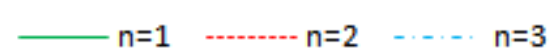

Figure 6. Normalized meridional vibration modes $n=1,2,3$ for the circumferential modes (a) $m=1$ (b) $m=2$ (c) $m=3$ (d) $m=4$ (e) $m=5$ (f) $m=6$ (g) $m=7$ (h) $m=8$

As a parametric study the curvature of the tower is varied by altering the shape parameter, $\mathrm{K}$ by keeping throat radius constant with changing characteristic dimension, $b$. The shape parameter is altered between 1 and 2 . The height and wall thickness of the tower are also kept constant. The periods of first five modes of the cooling tower with variation of shape parameter $(\mathrm{K})$ are given in Table 4. 
Table 4. The periods of first five modes of the cooling tower with variation in meridional curvature or shape parameter, $\mathrm{K}$

\begin{tabular}{cccccc}
\hline $\begin{array}{c}\text { Shape } \\
\text { parameter, K }\end{array}$ & Mode1 & Mode2 & Mode3 & Mode4 & Mode5 \\
\hline 1.0 & 1.644 & 1.152 & 1.145 & 0.741 & 0.600 \\
1.2 & 0.685 & 0.622 & 0.545 & 0.540 & 0.520 \\
1.4 & 0.633 & 0.606 & 0.596 & 0.583 & 0.552 \\
1.6 & 0.686 & 0.684 & 0.678 & 0.664 & 0.629 \\
1.8 & 0.787 & 0.776 & 0.774 & 0.744 & 0.735 \\
2.0 & 0.891 & 0.887 & 0.865 & 0.857 & 0.810 \\
\hline
\end{tabular}

It can be seen from Figure 7 that increasing curvature causes the first five periods to decrease first until a certain value whereupon periods increase. This behavior is observed strongest for the fundamental period of

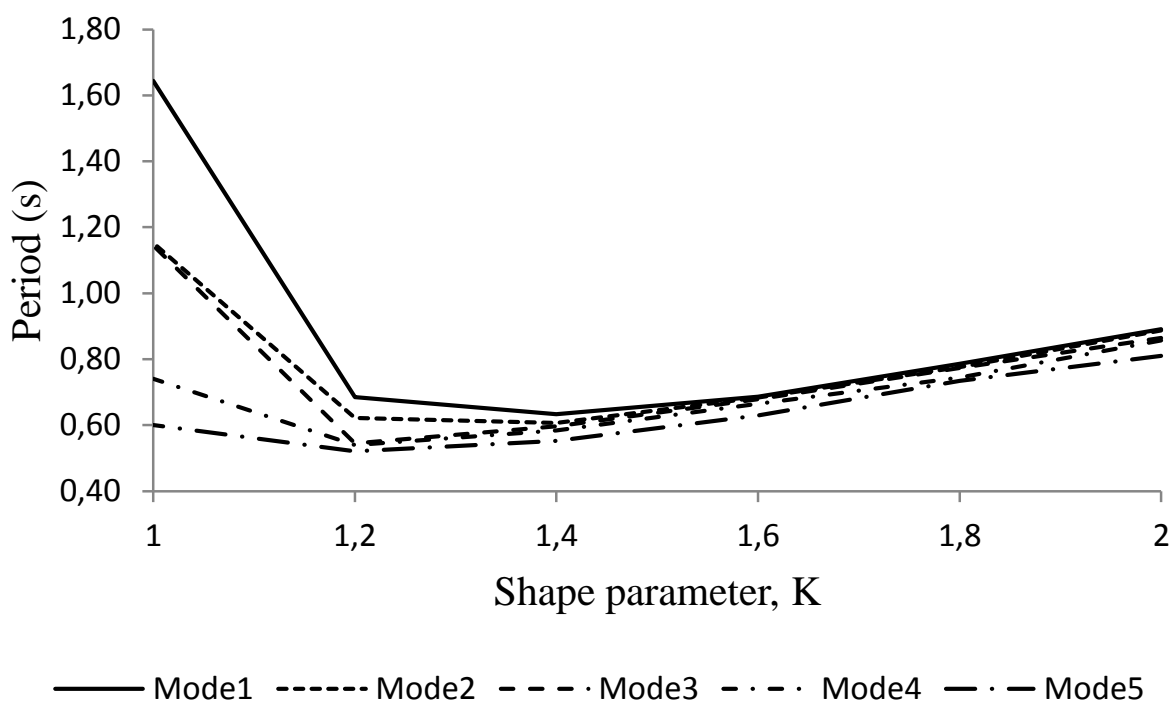

Figure7. The influence of shape parameter $\mathrm{K}$ on the periods of first five of modes

\subsection{Dynamic Analysis of the Cooling Tower}

Time-dependent seismic actions are the only dynamic load of interest in this part. The load is modeled in the finite element time history analysis using recorded ground accelerations and the responses are obtained by Newmark direct integration method. The transient responses of the four cooling tower structures with variations in throat diameters as given in Table 5 are investigated for meridional and circumferential stresses. The throat diameter is altered between $55.78 \mathrm{~m}$ and $40.78 \mathrm{~m}$ and the shape of towers are shown in Table 5. All other parameters are vibration. Therefore, it can be said that the meridional curvature can significantly influence free vibration response of the tower. 
Table 5. Four different models analyzed for earthquake loading

\begin{tabular}{ccccc}
\hline Model & K1 & K2 & K3 & K4 \\
\hline $\begin{array}{c}\text { Throat } \\
\text { diameter }(\mathrm{m})\end{array}$ & 55.78 & 50.78 & 45.78 & 40.78 \\
\hline & & & & \\
& & & & \\
$\begin{array}{c}\text { Elevation } \\
\text { Views }\end{array}$ & & & & \\
& & & & \\
\hline
\end{tabular}

diameter decreases. Moreover, the maximum circumferential stress increases with increase in curvature while the maximum meridional stress decreases. Thus, it can be concluded that the hyperbolic geometry has a significant influence on the earthquake stress response that can be optimized by changing curvature of the cooling tower.

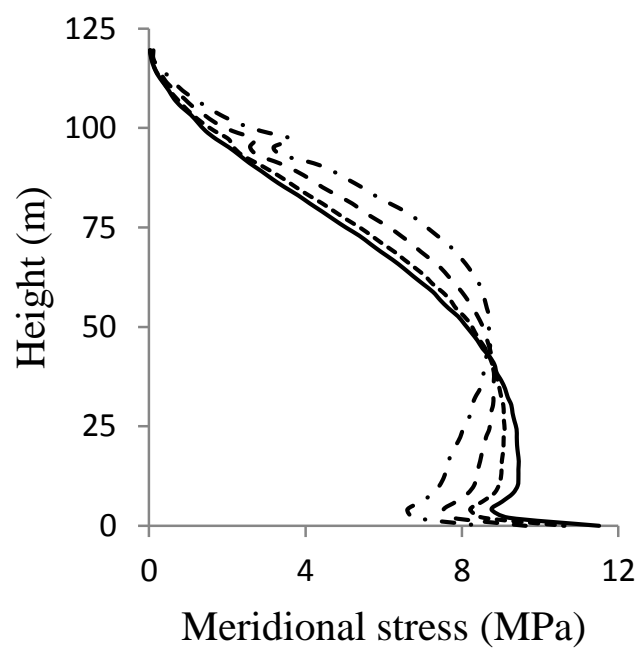

(a)

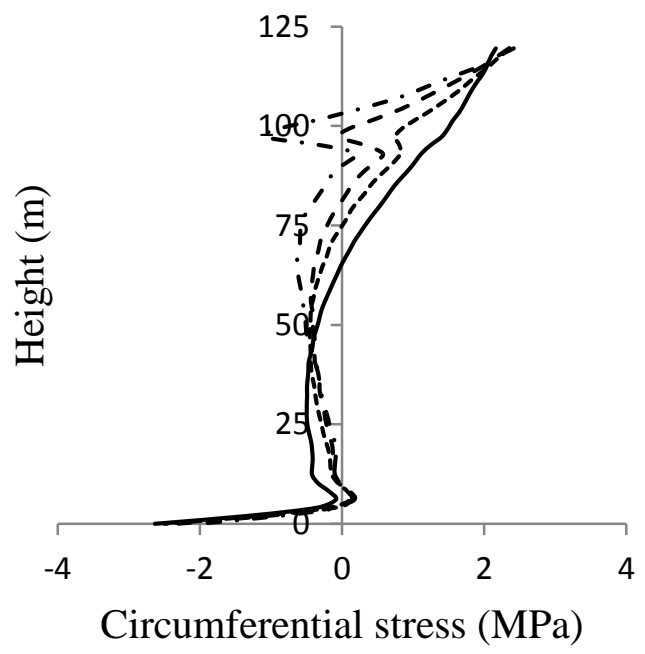

(b)

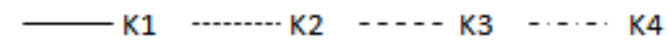

Figure 8. (a) Meridional and (b) circumferential stresses for along the height of the cooling towers with different curvatures when the maximum values are reached at $\theta=0$ meridian.

Figure 9(a, b) show the meridional and circumferential stress responses along the height of the tower at $\theta=0$ for each time step during Newmark dynamic analysis. During the earthquake these stresses change sign. In other words, they carry on reversing from tension to compression and vice versa as shown in Figure $9(\mathrm{a}, \mathrm{b})$. Dark regions in these figures indicate that the stresses intensify within small interval during the earthquake. 




Meridional stress (x10 MPa)

(a)

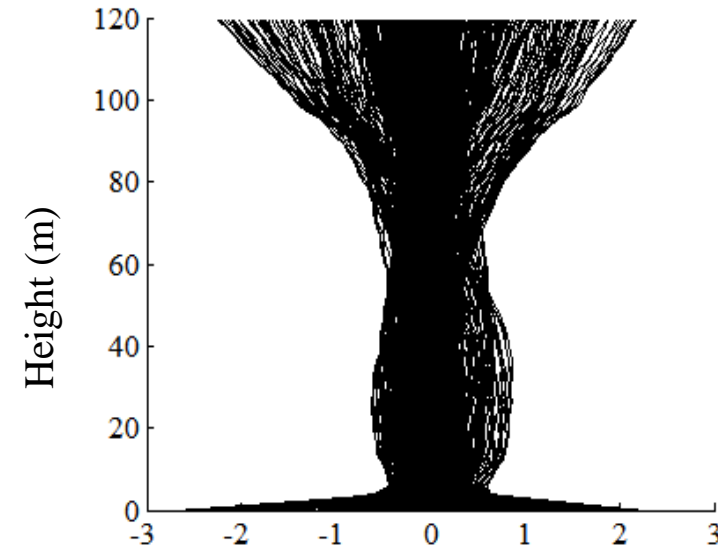

Circumferential stress (MPa)

(b)

Figure 9. a) Meridional and b) circumferential stresses along the height of the hyperbolic cooling tower at $\theta=0^{\circ}$ meridian during Düzce earthquake for each time step.

Figure $10(\mathrm{a}, \mathrm{b}, \mathrm{c})$ depict the time histories of the maximum lateral (radial) displacement at the top, maximum meridional stress at the base and maximum circumferential stress at the top of the towers with the smallest and largest throat diameter examined, respectively.As shown the maximum values during earthquake are obtained at around the time of maximum ground acceleration appeared at the 4.135 second of the record considered. The maximum radial displacement, tensional meridional and circumferential stresses are obtained as 63 $\mathrm{mm}, 11506 \mathrm{kPa}$ and $2300 \mathrm{kPa}$ while $67 \mathrm{~mm}$, $8718 \mathrm{kPa}$ and $2417 \mathrm{kPa}$ respectively for the tower with the smallest throat diameter.

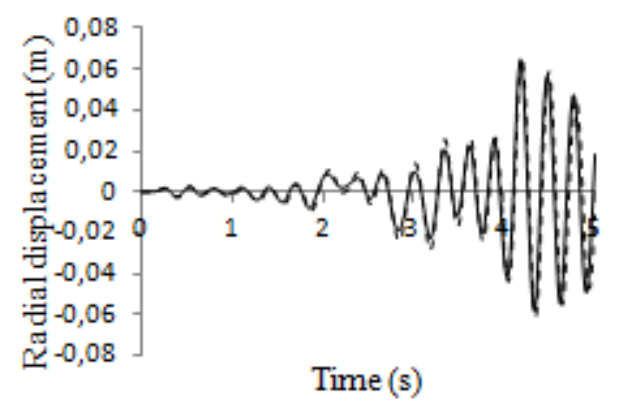

(a)

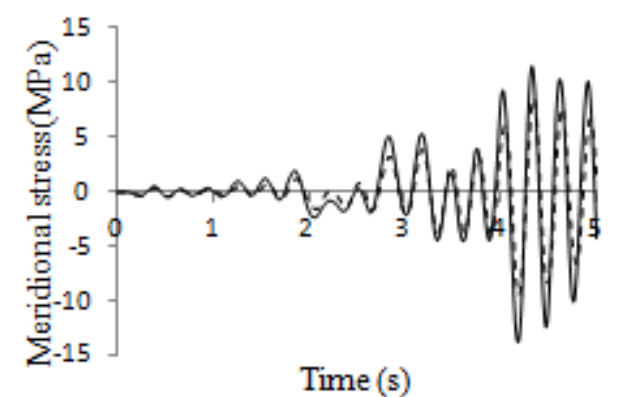

(b)

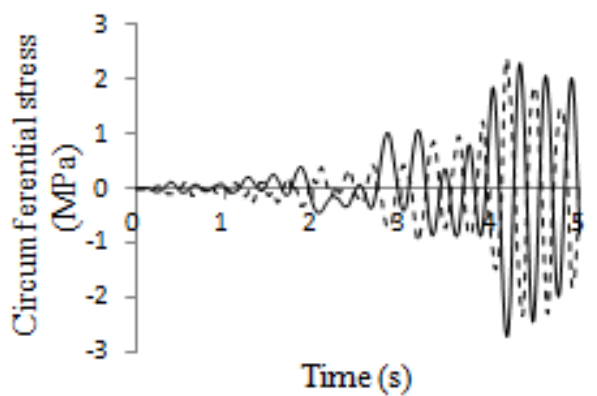

(c)

Figure 10. Time history of the maximum(a)lateral (ra dial) displacement (b)meridional and (c) circumferential stresses of the cooling tower at $\theta=0$ meridian during the earthquake. 


\section{Conclusions}

Free vibration characteristics and earthquake responses of a cooling tower are investigated using harmonic ring finite elements. Additionally, the influence of curvature on the periods and seismic responses of the tower are examined by changing throat diameter while keeping the other parameters constant. Most important conclusions drawn from the study are as follows:

- The time required for the dynamic analysis decreases extremely due to harmonic analysis by considering tower system as two-dimensional.

- The frequency of lateral mode of the cooling tower can be found easily without a complete free vibration analysis by defining circumferential mode number only.

- The natural frequencies of the cooling tower decrease with increasing circumferential mode number up to a minimum value after this they increase.

- Increasing curvature by decreasing throat diameter causes the fundamental periods to decrease until a certain value of curvature whereupon they increase.

- Hoop stresses at the throat level of the cooling tower are quite sensitive to the changes in curvature.

- Maximum circumferential and meridional stresses are observed at the top and at the base of the cooling tower under seismic loading, respectively.

\section{Acknowledgments}

The first author has been supported by TUBITAK (Turkish Scientific and Technological Research Council) with a scholarship which is gratefully acknowledged.

\section{References}

[1] Hashish MG, Abu-Sitta SH. Free vibration of hyperbolic cooling towers. J Eng Mech Div ASCE 1971; 97: 253269.

[2] Prasahanth N, Sayeed S. To study the effect of seismic loads and wind load on hyperbolic cooling tower of varying dimensions and rcc shell thickness. Int $\mathbf{J}$ Emerg Trends Eng Development 2013; 4(3): 260-269.

[3] Nasir AM, Thambiratnam DP, Butler D, Austin P. Dynamics of axisymmetric hyperbolic shell structures. Thin-Walled Struct 2002; 40: 665-690.

[4] Sam-Young N, Sang-Yun L. Evaluation of the natural draught cooling tower shell using linearly and non-linearly numerical analysis. J Vibroengineering 2012; 14(3): 1011-1020.

[5] Sam-Young N, Sang-Yun L. Structural behaviour evaluation of natural draught cooling towers under the consideration of shell-geometric parameters. Appl Mech Mater 2013; 284: 1396-1400.

[6] Calladine CR. Natural frequencies of cooling tower shells. J Sound and Vibration 1982; 82(3): 345-369.

[7] Tande SN, Snehal SC. Linear and Nonlinear Behavior of RC Cooling tower under earthquake loading. Int $\mathbf{J}$ Latest Trends in Eng Tech (IJLTET) 2013; 2(4): 370-379.

[8] Kulkarni S, Kulkarni AV. Static and dynamic analysis of hyperbolic cooling tower. J Civil Eng Tech Research 2014; 2 (1): 39-61.

[9] Cook RD, Malkus DS, Plesha ME. Concepts and Applications of Finite Element Analysis. $3^{\text {rd }}$ Edition, USA, Wiley \& Sons, 1989.

[10] Karakaş Aİ. Static and dynamic analyses of axisymmetric structures using harmonic rolid ring finite element modeling. Msc. Karadeniz Technical University, Trabzon, Turkey, 2012. 
[11] Benasciutti D, De Bona F, Munteanu MGh. A Semi-Analytical Finite Element Approach in Machine Design of Axisymmetric Structures, Numerical Analysis - Theory and Application Numerical Analysis-Theory and Application. Jan Awrejcewicz (Ed.), InTech, 2011.

[12] Bathe KJ. Finite Element Procedures. $1^{\text {th }}$ Edition, USA, Prentice-Hall, 1996.

[13] Bhatti MA. Advanced Topics in Finite Element Analysis of Structures: with Mathematica and Matlab Computations, USA, John Wiley\&Sons, 2006.
[14] Karadeniz H. Spectral Analysis Program of Structures: SAPOS User Manual, Delft University of Technology, Netherlands, 79-90, 2009.

[15] Smith, JW. Vibration of Structures: Applications in Civil Engineering Design, USA, Chapman and Hall, 1988.

[16] Wilson EL. Three-Dimensional Static and Dynamic Analysis of Structures, 3rd Edition, Berkeley, California, USA, Computers and Structures Inc.,2002.

[17] Gould PL, Kratzig WB. Handbook of Structural Engineering, $2^{\text {nd }}$ Edition, Germany, CRC Press, 2005. 